Muro de la Investigación, 2020(1), enero-junio

ISSN: 2523-2886

Doi: https://doi.org/10.17162/rmi.v5i1.1301

\title{
Uso académico de WhatsApp en grupos de estudiantes de ingeniería y arquitectura de una universidad privada
}

\section{Academic use of WhatsApp in groups of engineering and architecture students from a private university}

\author{
Augusto Lynch Becilla ${ }^{1 a}$, Jose Calsín Molleapaza ${ }^{2}$ \\ Universidad Peruana Unión, Lima, Perú ${ }^{12}$
}

Recibido: 12 de octubre de 2019

Aceptado: 15 de diciembre de 2020

\section{Resumen}

El objetivo de la presente investigación fue determinar el uso de la aplicación WhatsApp en grupo en estudiantes de la Facultad de Ingeniería y Arquitectura de la Universidad Peruana Unión (UPeU), Lurigancho-Chosica, Lima, 2018. La investigación es de tipo básica con enfoque cuantitativo, de diseño no experimental y descriptivo aplicado a una muestra de 304 estudiantes universitarios. Para este estudio se aplicó un cuestionario estandarizado para "WhatsApp en la regulación de trabajo en grupo", con cuatro dimensiones y 59 ítems, los cuales fueron adaptados y validados por juicio de expertos para evaluar la claridad, dominio de constructo, congruencia y contexto. Los resultados del análisis evidencian que el 68,8\% de los estudiantes poseen un nivel alto en el uso de la aplicación WhatsApp en grupos académicos. Asimismo, en cuanto a las dimensiones de uso de WhatsApp para trabajos grupales, el 67,6\% de los estudiantes evidenciaron un nivel alto: 79,8 \% como sistema de comunicación, $71,4 \%$ alto para las relaciones interpersonales, y 42,2\% manifiesta que el uso de WhatsApp para trabajos académicos presenta limitaciones. Se concluye que los estudiantes de la Facultad de Ingeniería y Arquitectura de la Universidad Peruana Unión del año 2018, poseen un alto nivel de uso de la aplicación WhatsApp para trabajos en grupos, lo cual representa una herramienta potencial para mejorar y aprovechar en el proceso de comunicación con fines académicos.

Palabras claves: WhatsApp, redes sociales, grupos académicos, relaciones interpersonales, comunicación digital.

\begin{abstract}
The objective of this research was to determine the use of the WhatsApp application in groups of students from the Faculty of Engineering and Architecture of the Peruvian Union
\end{abstract}

\footnotetext{
${ }^{\text {a}}$ Correspondencia a los autores

e-mail: augustolynch@upeu.edu.pe,josecalsin@upeu.edu.pe
} 
University (Universidad Peruana Union, UPeU), in the district of Lurigancho-Chosica, Lima, 2018. The research is of a basic type with a focus quantitative, non-experimental and descriptive design applied to a sample of 304 university students. For this study, a standardized questionnaire for "WhatsApp in the regulation of group work" was applied, with four dimensions and 59 items, which were adapted and validated by experts judgment to evaluate clarity, construction domain, congruence and context. The results of the analysis show that $68.8 \%$ of the students have a high level of use of the WhatsApp application in academic groups. Also, regarding the dimensions of use of WhatsApp for group work, 67.6\% of the students showed a high level: $79.8 \%$ as a communication system, $71.4 \%$ high for interpersonal relationships, and 42, $2 \%$ state that the use of WhatsApp for academic works presents limitations. In conclusion, the students of the Faculty of Engineering and Architecture of the Universidad Peruana Unión of the year 2018 have a high level of use of the WhatsApp application for group work, which represents a potential tool to improve and take advantage of the process of communication for academic purposes.

Keywords: WhatsApp, social networks, academic groups, interpersonal relationships, digital communication.

\section{Introducción}

En los últimos años, los medios tecnológicos han revolucionado las formas de comunicación. La aparición de la redes sociales, destacando el uso del WhatsApp desde el 2009, un aplicativo móvil de mensajería instantánea, se ha convertido en todo un fenómeno social, por el cual se puede interactuar compartiendo contenidos audiovisuales, lingüísticos y otros (Ramos, 2015). Al respecto, se estima que en el 2019, la población activa del WhatsApp será de 1700 millones (Androitphonia, 2018). Este escenario, plantea algunas preguntas con respecto a las dinámicas de vida en la sociedad de la información, pues, es evidente cómo cada vez más, las personas están interconectadas.

$\mathrm{Al}$ respecto, la integración de la tecnología en la vida cotidiana — en especial en la generación de los millennials (personas nacidas entre 1980 y 2000) — ha generado un cambio en las formas de vida, desde las actividades realizadas de manera individual hasta las acciones realizadas en lo colectivo. Estas maneras de vivir se hacen evidentes en las organizaciones, el entretenimiento, el trabajo, la escuela, entre otros (Gutiérrez-Rubí, 2015). En este contexto, los móviles se han convertido en una extensión de las habilidades del ser humano, pues posibilitan y facilitan acciones de comunicación en el tiempo y espacio.

Un estudio de Tomi Alhonen-citado por Gutiérrez-Rubí (2015)—señala que los usuarios revisan su móvil en un promedio de 150 veces por día. Este comportamiento social se hace cada vez más latente con la aparición de nuevos aplicativos y mejores equipos, lo 
cual hace recurrente la necesidad de integrarlo en temas prioritarios como la educación. Según Celaya et al. (2015) existen tres ámbitos donde ocurre una buena comunicación: la vida en común, la convivencia y el aprendizaje, siendo este último el interés prioritario de la presente investigación.

En el contexto educativo, la integración de las tecnologías de la información (TICs) es ya una realidad (Fuentes et al., 2017); sin embargo, existe una amplia brecha para nivelar los avances a las metodologías de enseñanza. Los estudiantes de la generación millennial han experimentado un cambio radical, son nativos digitales que dominan la utilización y dinámica comunicacional de las redes, lo cual ha generado numerosas modificaciones en las formas de pensar, procesar y entender la realidad en el alumnado (Prensky, 2010). En este escenario, los docentes enfrentan un gran desafío, pues tienen dificultades en entender las diferencias y aprender a comunicarse con los estudiantes en su estilo y al ritmo que ellos manejan.

No obstante, en toda esta vorágine comunicativa, los estudiantes hacen uso de las redes sociales para las actividades académicas, siendo la aplicación WhatsApp la más usada para coordinar actividades grupales en estudiantes (Bouhnik y Deshen, 2014). Algunas investigaciones (Cifuentes y Lents, 2010) afirman que la aplicación ayuda a que el alumnado sea más activo, y se produzca una expansión de aprendizaje más allá del aula (Fewkes y McCabe, 2012). Por otro lado, las limitaciones que enfrentan están asociadas a cuestiones de privacidad y distracción durante el desarrollo de la clase (Wang et al, 2012), pues dificulta a los docentes implementar la aplicación adecuadamente en la enseñanza (Fewkes y McCabe, 2012).

Los docentes son responsables del aprendizaje de los estudiantes, los cuales, “...esperan que sus profesores sepan más de lo que ellos saben y que sepan cómo ayudarlos a adquirir más conocimientos de ellos mismos. Esperan que los adultos, que son responsables de ellos, sepan cómo ayudarlos a ir a donde no pueden ir por sí mismos" (Abril y Villa lba, 2014). Asimismo, "el profesor ya no puede presentarse como el dueño exclusivo del conocimiento, sino más bien como quien ayuda al estudiante a que este construya su propio conocimiento" (Cortés Marín, 2007). En tal sentido, las competencias que el docente tenga 
que nivelar para la enseñanza, posibilita una experiencia de aprendizaje satisfactoria y significativa en los estudiantes, lo cual repercute en otros ámbitos de su vida.

De la misma forma, la importancia de una retroalimentación sobre las experiencias de aprendizaje contribuye en las experiencias del estudiante, pues permite generar información sobre la efectividad de la enseñanza de los docentes, adquirir información para la toma de decisiones respecto a los métodos de enseñanza, y obtener datos para ser usados en investigaciones sobre los procesos de enseñanza/aprendizaje (González, López, \& Montenegro, 2012)

Por lo expuesto, el estudio del uso académico del WhatsApp en grupos contribuirá a la generación de conocimientos que mejoren los procesos comunicativos en el área de la educación, que genere una mejor experiencia para el estudiante como para el docente, utilizando metodologías que faciliten el acceso a la información y la comunicación acortando las distancias y optimizando la calidad de tiempo. En este sentido, el presente estudio tiene por objetivo determinar el uso académico de la aplicación WhatsApp en grupo en estudiantes de ingeniería de la Universidad Peruana Unión, 2018. Asimismo, pretende ahondar aspectos poco explorados desde el enfoque de la educomunicación, haciendo una revisión de autores

y teorías de área, a partir de un enfoque de las tecnologías de la comunicación (TICs) y su integración con el proceso de enseñanza aprendizaje. Todo esto implica responder la siguiente pregunta: ¿En qué medida usan académicamente la aplicación WhatsApp los grupos de estudiantes de ingeniería de la Universidad Peruana Unión, 2018?

\section{Metodología}

Tipo de investigación

La presente investigación es de tipo básica, pues pretende ampliar los conocimientos (Bunge, 1978) en relación al uso de la aplicación WhatsApp en grupos de estudiantes de ingeniería de la Universidad Peruana Unión, 2018.

Diseño de investigación

La presente investigación es de diseño no experimental, porque no se manipulará deliberadamente la variable "uso de la aplicación WhatsApp en grupos", y se observará el comportamiento de la misma tal y como se da en su estado natural. Por otro lado, es transversal, ya que los datos se recolectarán en un tiempo único, en la fecha establecida por 
el investigador. Por último, es descriptiva, pues se describirá los usos de la aplicación WhatsApp en grupo de estudiantes de ingeniería de la Universidad Peruana Unión en el año 2018.

Población y muestra

La población de la presente investigación consiste en 1461 estudiantes de la Facultad de Ingeniería y Arquitectura de la Universidad Peruana Unión de Ñaña, ubicada en la Carretera Central km. 19, Ñaña, Lurigancho-Chosica, en el departamento de Lima. De la cual, con el método de poblaciones finitas con un nivel de confianza de $95 \%$ y un 5\% de margen de error, se extrajo una muestra de 304 estudiantes a quienes será aplicado el cuestionario.

Técnica de recolección de datos

Para el desarrollo de este estudio se tomará un cuestionario que mide la variable: "uso de WhatsApp en grupo de estudiantes universitarios en el año 2018”, lo cual será sometido a una validez de contenido por juicio de expertos, a fin de ver el contexto y la pertinencia de los factores medibles. Posteriormente se administrará una prueba piloto a un grupo de estudiantes universitarios de la Facultad de Ingeniería y Arquitectura de la Universidad Peruana Unión.

Con el instrumento listo y validado se presentará la documentación respectiva al comité de ética de la Universidad Peruana Unión y se solicitará permiso a la Dirección General de Investigación para desarrollar el estudio. Luego, se les explicará a los estudiantes el llenado debido del cuestionario. Finalmente, el procesamiento de los datos se gestionará con el programa estadístico IBM-SPSS, con el cual se vaciarán los datos obtenidos. Posteriormente, se analizarán e interpretarán los datos para la elaboración del informe final y la discusión de resultados.

Fiabilidad del Instrumento

El análisis de fiabilidad del instrumento "Uso de WhatsApp para la regulación de trabajo en grupo de estudiantes universitarios" tiene un valor Alfa de Cronbach es de 0,951; este resultado evidencia que el instrumento tiene un grado elevado de confiabilidad. Asimismo, en el análisis de fiabilidad de las dimensiones, los resultados muestran que el 
valor Alfa de Cronbach de las dimensiones $1(0,894)$ y $2(0,893)$ tienen un grado de fiabilidad aceptable, mientras que el valor de las dimensiones $3(0,936)$ y $4(0,906)$ tienen un grado de fiabilidad elevada. En el coeficiente de confiabilidad, se determina que el instrumento de medición se aproxima a ser de consistencia interna "elevada".

\section{Resultados}

En la tabla 1 se observa que el 51,0\% de los estudiantes que participaron del estudio son varones y el 49,0 \% son mujeres.

Tabla 1

Sexo de los estudiantes

\begin{tabular}{lcc}
\hline Sexo & Frecuencia & Porcentaje \\
\hline Hombre & 152 & 51.0 \\
Mujer & 146 & 49.0 \\
\hline Total & $\mathbf{2 9 8}$ & $\mathbf{1 0 0 . 0}$ \\
\hline
\end{tabular}

Fuente: Elaboración propia

En la tabla 2 se aprecia que la edad del 58,6\% de los estudiantes objetos de estudio oscila entre 17 y 20 años; el 36,9 \% entre 21 y 25 años; el 3,0 \% más de 25 años y el 1,4 \% menos de 16 años.

Tabla 2

Edad de los estudiantes

\begin{tabular}{lcc}
\hline \multicolumn{1}{c}{ Edad } & Frecuencia & Porcentaje \\
\hline Menos de 16 años & 4 & 1.4 \\
De 17 a 20 años & 173 & 58.6 \\
De 21 a 25 años & 109 & 36.9 \\
Más de 25 años & 9 & 3.1 \\
\hline Total & $\mathbf{2 9 5}$ & $\mathbf{1 0 0 , 0}$ \\
\hline
\end{tabular}

Fuente: Elaboración propia

La tabla 3 muestra que el 40, $0 \%$ de los estudiantes pertenecen a la Escuela Profesional de Ingeniería Ambiental; el 22,0 \% a Arquitectura; el 16,8 \% a Ingeniería de Sistemas; el 11,5\% a Ingeniería Civil y el 8,9 \% a Ingeniería de Alimentos. 
Tabla 3

Escuela Profesional a la que pertenecen los estudiantes

\begin{tabular}{lcc}
\hline \multicolumn{1}{c}{$\begin{array}{c}\text { Escuela } \\
\text { Profesional }\end{array}$} & Frecuencia & Porcentaje \\
\hline Ingeniería & & \\
Ambiental & & 40.8 \\
Ingeniería & & 16.8 \\
Sistemas & 124 & 8.9 \\
Ingeniería de & 51 & 22.0 \\
Alimentos & 27 & 11.5 \\
Arquitectura & 67 & \\
Ingeniería Civil & 35 & \\
\hline Total & $\mathbf{3 0 4}$ & $\mathbf{1 0 0 , 0}$ \\
\hline
\end{tabular}

Fuente: Elaboración propia

En la tabla 4 se aprecia que el $54,1 \%$ de los estudiantes utilizan equipos de gama media; el $20,5 \%$ de gama alta y el $10,3 \%$ de gama baja.

Tabla 4

Tipo de gama de celular

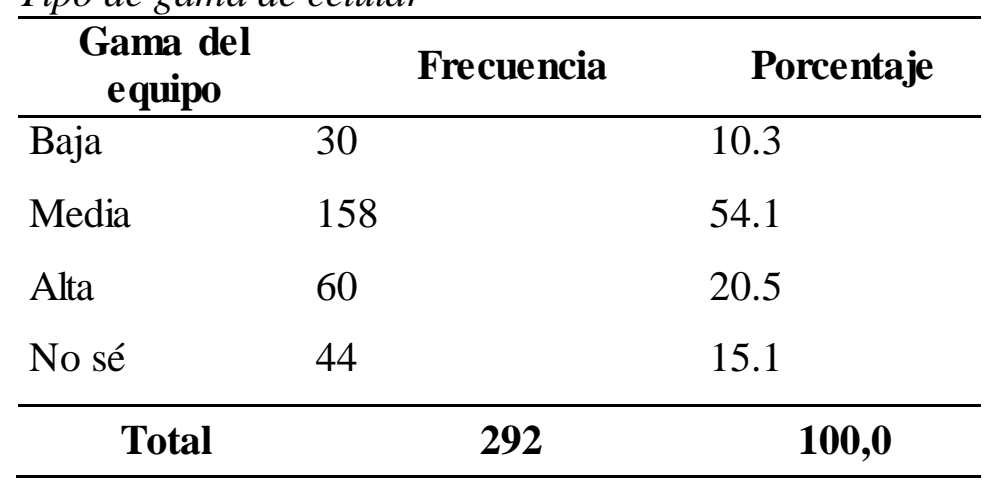

Fuente: Elaboración propia

La tabla 5 evidencia que el 69,6\% de los estudiantes manifiestan pertenecer a tres grupos académicos de WhatsApp, mientras que el 29,7 declaran pertenecer a dos grupos y el $0,3 \%$ expresan pertenecer a uno y cuatro grupos académicos de WhatsApp. 
Tabla 5

Grupos académicos de WhatsApp a los que pertenece el estudiante

\begin{tabular}{|c|c|c|}
\hline $\begin{array}{l}\text { Grupos de } \\
\text { WhatsApp }\end{array}$ & Frecuencia & Porcentaje \\
\hline Uno & 1 & 0.3 \\
\hline Dos & 87 & 29.7 \\
\hline Tres & 204 & 69.6 \\
\hline Cuatro & 1 & 0.3 \\
\hline Total & 293 & 100,0 \\
\hline
\end{tabular}

Fuente: Elaboración propia

En la tabla 6 se observa que el $42,44 \%$ de los estudiantes declaran revisar su WhatsApp tres o cuatro veces por semana, el 31,0\% hace todos los días, el 22,2\% una o dos veces por semana y el $4,4 \%$ manifiesta nunca revisarlo.

Tabla 61

Frecuencia de tiempo del uso de WhatsApp

\begin{tabular}{lcc}
\hline Revisar los mensajes & $\begin{array}{c}\text { Frecuen } \\
\text { cia }\end{array}$ & Porcentaje \\
\hline Todos los días & 92 & 31.0 \\
3 o 4 veces a la semana & 126 & 42.4 \\
1 o 2 veces por semana & 66 & 22.2 \\
Nunca & 13 & 4.4 \\
\hline Total & $\mathbf{2 9 7}$ & $\mathbf{1 0 0 , 0}$ \\
\hline
\end{tabular}

Fuente: Elaboración propia

La tabla 7 evidencia que el 68,8 \% de los estudiantes poseen un nivel alto en el uso de la aplicación WhatsApp en grupos académicos, mientras que el 31,2 \% tienen un nivel medio en el uso de la aplicación. 
Tabla 7

Niveles de uso de la aplicación WhatsApp en grupos

\begin{tabular}{lcc}
\hline \multicolumn{1}{c}{ Nivel } & Frecuencia & Porcentaje \\
\hline Medio & 79 & 31.2 \\
Alto & 174 & 68.8 \\
\hline Total & $\mathbf{2 5 3}$ & $\mathbf{1 0 0 , 0}$ \\
\hline
\end{tabular}

Fuente: Elaboración propia

La tabla 8 muestra que el 67,6 \% de los estudiantes posee un nivel alto en el uso de la aplicación WhatsApp para la organización de trabajos grupales, mientras que el 30,6 \% posee un nivel medio de uso y finalmente el 1,8\% tiene un nivel bajo en el uso de la aplicación para la organización de trabajos grupales.

Tabla 8

Uso de la aplicación WhatsApp para la organización de trabajos grupales

\begin{tabular}{lcc}
\hline \multicolumn{1}{c}{ Nivel } & Frecuencia & Porcentaje \\
\hline Bajo & 5 & 1.8 \\
Medio & 87 & 30.6 \\
Alto & 192 & 67.6 \\
\hline Total & $\mathbf{2 8 4}$ & $\mathbf{1 0 0 , 0}$ \\
\hline
\end{tabular}

Fuente: Elaboración propia

La tabla 9 demuestra que el 79,8\% de los estudiantes tiene un nivel alto en el uso de la aplicación WhatsApp como sistema de comunicación de tareas grupales, mientras que el $18,9 \%$ tiene un nivel medio de comunicación y el 1,3\% tiene un nivel bajo en el uso de la aplicación para la comunicación de tareas grupales.

Tabla 92

Uso de la aplicación WhatsApp como sistema de comunicación

\begin{tabular}{lcc}
\hline \multicolumn{1}{c}{ Nivel } & Frecuencia & Porcentaje \\
\hline Bajo & 4 & 1.3 \\
Medio & 56 & 18.9 \\
Alto & 237 & 79.8 \\
\hline Total & $\mathbf{2 9 7}$ & $\mathbf{1 0 0 , 0}$ \\
\hline
\end{tabular}

Fuente: Elaboración propia 
En la tabla 10 se observa que el 71,4 \% de los estudiantes posee un nivel alto en el uso de la aplicación WhatsApp para las relaciones interpersonales; el 27,6 \% hace uso de un nivel medio y el 1,0\% tiene un nivel bajo en el uso de la aplicación para para las relaciones interpersonales.

Tabla 30

Uso de la aplicación WhatsApp para las relaciones interpersonales

\begin{tabular}{lcc}
\hline Nivel & Frecuencia & Porcentaje \\
\hline Bajo & 3 & 1.0 \\
Medio & 80 & 27.6 \\
Alto & 207 & 71.4 \\
\hline Total & $\mathbf{2 9 0}$ & $\mathbf{1 0 0 , 0}$ \\
\hline
\end{tabular}

Fuente: Elaboración propia

En la tabla 11, el 53,3\% de los estudiantes, declaran el uso de la aplicación WhatsApp presenta un nivel medio en la limitación para trabajar en grupos, el 42,2 \% manifiestan que presenta un nivel alto de limitación y el 4,5 \% revelan que presenta un nivel bajo de limitación para trabajar en grupos.

Tabla 11

Uso de la aplicación WhatsApp presenta limitaciones para trabajar en grupos

\begin{tabular}{lcc}
\hline Nivel & Frecuencia & Porcentaje \\
\hline Bajo & 13 & 4.5 \\
Medio & 154 & 53.3 \\
Alto & 122 & 42.2 \\
\hline Total & $\mathbf{2 9 7}$ & $\mathbf{1 0 0 , 0}$ \\
\hline
\end{tabular}

\section{Discusión}

El principal objetivo de este estudio fue determinar el uso de WhatsApp en grupos académicos de estudiantes de ingeniería y arquitectura de la Universidad Peruana Unión, mediante un instrumento que permitió medir los niveles de uso de la aplicación referida en grupos académicos. Al analizar los resultados en relación al objetivo general, la investigación 
evidenció que el 96\% de los jóvenes de la Facultad de Ingeniería y Arquitectura utilizan la red social WhatsApp; 54\% de ellos poseen equipos de celular de gama intermedia, lo que implica: que los estudiantes cuentan con celulares con pantallas amplias y buena resolución, una cámara que permite tomar fotografías con una resolución buena o intermedia, y características que permiten el uso de la aplicación WhatsApp con total comodidad.

Asimismo, en este estudio se evidencia que la frecuencia diaria de uso de WhatsApp es de 31\%; el $42 \%$ lo usa 3 o 4 veces, y el 22\% 1 o 2 veces por semana; tal situación corrobora que el $96 \%$ de los estudiantes cuentan con acceso al Whatsapp, aunque en diferentes grados de frecuencia de uso. Por otro lado, el $68 \%$ de los estudiantes presenta en un nivel alto de uso de WhatsApp en grupos académicos, y $31 \%$ en un nivel medio. $67 \%$ de los estudiantes utilizan el WhatsApp en grupos académicos para la organización de trabajos grupales; el 79\% lo usa como sistema de comunicación, y el $71 \%$ de los estudiantes considera que su uso mejora las relaciones interpersonales. Esta situación corrobora lo que mencionan Santa-Cruz y Villareal (2017), que el aprendizaje está ligado al uso de las tecnologías de la comunicación. Al respecto, Serra et al., (2018) encontraron en su investigación que el medio más utilizado por jóvenes estudiantes en una universidad del Ecuador es el Whatsapp en un $99.5 \%$, dato similar al que encontraron Rubio \& Perlado (2015), quienes mencionan que el uso de WhatsApp se adapta mejor al uso y la movilidad práctica. De otro lado, Padrón (2013) encontró que el uso de Whatsapp en la educación, integrado a la estrategia formativa, permite la construcción del conocimiento entre grupo y mejora la comunicación.

De igual forma Giasanti et al., (2016) consideran que el uso de la aplicación WhatsApp es útil e importante en el acceso a la información y el apoyo al proceso de aprendizaje. Estos resultados sustentan lo que encontró Gallegos y Vigil (2015), donde los estudiantes mencionan que el uso de las TICs se ha convertido en una necesidad elemental para el aprendizaje.

De otro lado, los resultados de este estudio muestran que el $53 \%$ de los estudiantes tienen limitaciones para trabajar en grupos. En ese sentido, Rodríguez et al., (2017) mencionan que tanto padres, autoridades y escolares deben trabajar en la autorregulación del uso del WhatsApp, eliminando los distractores y aprovechando mejor el tiempo para usos formativos. 


\section{Conclusiones}

De acuerdo a los resultados obtenidos a través del análisis estadístico, las conclusiones en función a los objetivos planteados son las siguientes:

- Respecto al objetivo general, los estudiantes de ingeniería de la Universidad Peruana Unión del año 2018, tienen un alto uso de la aplicación WhatsApp para trabajos en grupos.

- Con respecto al objetivo específico 1, los estudiantes de ingeniería de la Universidad Peruana Unión del año 2018, usan la aplicación WhatsApp en grupo en un nivel alto para la organización de trabajos de grupales.

- Asimismo, en el objetivo específico 2, los estudiantes de ingeniería de la Universidad Peruana Unión del año 2018, utilizan WhatsApp como sistema de comunicación para las tareas grupales.

- Igualmente, en el objetivo específico 3, los estudiantes de ingeniería de la Universidad Peruana Unión del año 2018, hacen uso en un nivel alto la aplicación del WhatsApp en grupo para mejorar las relaciones interpersonales.

- Finalmente, en el objetivo específico 4, los estudiantes de ingeniería de la Universidad Peruana Unión, 2018, declaran que el uso de la aplicación del WhatsApp presenta un nivel medio de limitaciones para el trabajo de grupo.

\section{Referencias}

Abril, M., \& Villalba, M. A. (2014). La educación literaria: experiencias de aprendizaje. Octaedro. Recuperado de https://books.google.com.pe/books?id=xAcsrgEACAAJ

Androitphonia. (2018). Número de usuarios que usa WhatsApp en 2018. Retrieved July 12, 2018, from https://androidphoria.com/curiosidades/usuarios- usan-whatsapp-2018

Bouhnik, D., \& Deshen, M. (2014). WhatsApp goes to school: mobile instant messaging between teachers and students. Journal of Information Technology Education: Research, $13 . \quad$ Recuperado de http//www.jite.org/documents/Vol13/JITEv13ResearchP217-231Bouhnik0601.pdf

Celaya, M., Chacón, A., Chacón, A., \& Urrutia, E. (2015). El impacto de WhatsApp en la vida cotidiana de las personas- ¿Hace la sociedad más humana? Eskibel, 16. 
Cifuentes, O. E., \& Lents, N. H. (2010). Increasing student-teacher interactions at an urban commuter campus through instant messaging and online office hours. Electronic Journal of Science Education, 14(1), 1-13.

Concepción Lira-Rodríguez, M., Olimpia Reyes-Hernández, P., Reyes-Gómez, U., PereaMartínez, A., Hernández-Lira, S., Lizeth Reyes-Hernández, K., Colón-Cuesta, F. (2017). ¿Es el WhatsApp un distractor en el rendimiento escolar de los estudiantes de medicina? Boletin clinico Hospital Infantil del Estado de Sonora, 34(1), 26-34. Recuperado de http//search.ebscohost.com/login.aspx?direct=true\&db=lth\&AN=123168626\&lang $=$ es $\&$ site $=$ eds-live $\&$ scope $=$ site $\&$ custid $=s 4608367$

Cortés Marín, E. A. (2007). El nuevo rol del docente universitario. Revista CES Medicina Veterinaria y Zootecnia, 2, 89-99.

Fewkes, A. M., \& McCabe, M. (2012). Facebook: learning tool or distraction? Journal of Digital Learning in Teacher Education, 28(3), 92-98. http//doi.org/10.1080/21532974.2012.10784686

Fuentes Gutierrez, V., García Domingo, M., \& Aranda López, M. (2017). Grupos de clase; grupos de whatsapp: análisis de las dinámicas comunicativas entre estudiantes universitarios. Red de revistas cientiúficas de América Latina y el Caribe, España y Portugal, 29.

Gallegos Zegarra, F. L. M., \& Vigil Cannon, S. S. (2015). Influencia de la mensajería instantánea en las actitudes y nivel de conocimiento de las TICs en los estudiantes de $5^{\circ}$ Año, de la especialidad de Relaciones Públicas de la Escuela de Ciencias de la Comunicación UNSA, 2015 (tesis de licenciatura). Universidad Nacional San Agustín. Recuperado de http//repositorio.unsa.edu.pe/bitstream/handle/UNSA/2162/CCgazeflm.pdf?sequen ce $=1 \&$ is Allowed $=y$

Giasanti Tavares, A. R. P., Taboada Sobral, A. P., \& Jansiski Motta, L. (2016). Uso de la aplicación Whatsapp por estudiantes de odontología de Sao Paulo, Brasil. Revista cubana de información en ciencias de la salud, 27(4), 503-514. Recuperado de http $/ /$ scielo.sld.cu/scielo.php?script=sci_arttext\&pid=S2307-21132016000400007

González, C., López, L., \& Montenegro, H. (2012). Análisis de confiabilidad y de validez 
del instrumento "Course Experience Questionnaire” (CEQ). Educación y educadores, 15(1), 63-78. Recuperado de http $/ /$ educacionyeducadores. unisabana.ed u.co/index.php/eye/article/view/1903/271 4

Gutiérrez-Rubí, A. (2015). La poítica en tiempos de Whatsapp. Catalunya: El Pais. Recuperado de https://play.google.com/books/reader?id=Obi3BwAAQBAJ \&printsec=frontcover \& output=reader\&hl=es_419\&pg=GBS.PP1

Padrón, C. J. (2013). Estrategias didácticas basadas en aplicaciones de mensajería instantánea Whatsapp exclusivamente para móviles y el uso de la herramienta para promover el aprendizaje colaborativo. Revista de tecnologia e información y comunicación en educación, $\quad 7(2), \quad$ 123-134. $\quad$ Recuperado de http //servicio.bc.uc.edu. ve/educacion/eduweb/v7n2/art09.pdf

Prensky, M. (2010). Nativos e inmigrantes digitales. Institución Educativa Sek, 23. Recuperado de https://www.marcprensky.com/writing/Prensky-NATIVOS E INMIGRANTES DIGITALES \%28SEK\%29.pdf

Ramos, J. (2015). Marketing con WhatsApp. Guía práctica. Berlin: GD Publishing Ltd. \& Co KG, Berlin. $\quad$ Recuperado de https://books.google.com.pe/books?id=LTr9CwAAQBAJ\&pg=PT26\&dq=whatsapp $\& h l=e s \& s a=X \&$ ved=0ahUKEwjvy62gpJjc AhWpwFkKHUD7 A7EQ6AEIQ zAE\#v= onepage $\& \mathrm{q}=$ whatsapp $\& \mathrm{f}=$ false

Rubio Romero, J., \& Perlado Lamo de Espinosa, M. (2015). El fenómeno WhatsApp en el contexto de la comunicación personal: una aproximación a través de los jóvenes universitarios. Revista ICONO14. Revista científica de comunicación y tecnologías emergentes, 13(2), 73. http://doi.org/10.7195/ri14.v13i2.818

Santa-Cruz Villareal, F. (2017). Uso de las tecnologías de la información y comunicaciones (TICS) y su relación con el aprendizaje de los alumnos, en la Institución Educativa Pedro Ruiz Chorrillos, 2017. Instituto Científico y Tecnológico del Ejercito. Recuperado de http//renati.sunedu.gob.pe/bitstream/sunedu/199893/1/TESIS SANTA CRUZ VILLARREAL USO DE LAS TICS Y EL APRENDIZAJE IE PRG 2017.pdf 
Serra, C., Martorell, C., Mantilla, J., Larrea, A. M., \& Mantilla, P. (2018). El uso académico de Facebook y WhatsApp en estudiantes universitarios: un estudio comparativo entre España y Ecuador. Ecos de La Academia, 6(February), 209.

Wang, Q., Woo, H. L., Quek, C. L., Yang, Y., \& Liu, M. (2012). Using the Facebook group as a learning management system: an exploratory study. British Journal of Educational Technology, 43(3), 428-438. http //doi.org/10.1111/j.14678535.2011.01195.x 